defined syllable divisions. The authors conclude that the machine production of Braille text is not an economic possibility at present, in view of the large amount of voluntary effort available.

Chapter 8 describes an experimental scheme for translating from French into English devised for APEXC. For reasons of storage space the scheme was limited to a dictionary of 250 words and their English equivalents, but the programme was sufficiently comprehensive to perform certain operations of syntactical nature, and to recognize the masculine and feminine, singular and plural forms of nouns and adjectives, and also all parts of the verb except the first person imperative and the subjunctive mood.

The last part of the book is devoted to the translation of German, a subject which, unfortunately, the reviewer is not able to comment on.

The authors conclude by considering the technical details of a machine designed specially for translating. The principal feature of such a machine, of course, is a large high-speed store. Various special orders are recommended to facilitate the dictionary look-up process, and at the same time orders such as multiplication can be dropped. The authors suggest that such a machine could be built for between $£ 50,000$ and $£ 100,000$ but are rather sceptical of obtaining financial support on this scale. However, their proposed instruction code for a linguistic machine is not so very special, and it would scarcely seem worth while to build a machine on this scale which would exclude it from doing tasks other than language translation. The authors' best hope, therefore, lies in the super-high-speed computers at present under development, such as Stretch or Larc.

R. A. Brooker

\section{WORK ON FUSED SALTS}

\section{Soviet Research in Fused Salts (1949-1955)}

(Chemistry Collection, No. 1.) General. Pp. 28. Structure and Properties: Ternary Reciprocal. Pp. 200. Structure and Properties: Quaternary Systems; Quaternary Reciprocal Systems. Pp. 98. Structure and Properties: Preparation; Iodine Systems. Pp. 156. Structure and Properties: Binary Systems: Ternary Systems. Pp. 284. Slags and Mattes. Pp. 39. Electrochemistry. Pp. 54. Thermodynamics. Pp. 58. Translated from the Russian. (New York : Consultants Bureau, Inc.) n.p.

TNTEREST in the structure and properties of fused salts has been growing with noteworthy vigour in the past few years in various countries. From the fundamental point of view, molten salts constitute a distinct class of fluids, with an extensive range of temperatures over which the liquid state may be stable, with high heats of vaporization, and with quasi-crystalline structures in which the tendency for an ion of one sign to be preferentially surrounded by ions of opposite sign imposes an additional element of order, compared with other liquids. Despite the technical difficulties to be overcome, these characteristic properties have stimulated various basic researches on the transport and thermodynamic properties of ionic melts, and on melting mechanisms of ionic crystals.

The very great importance of fused salts in electrometallurgy, particularly of the more electropositive or reactive cation-forming elements, has for many years prompted applied research the bulk of which greatly exceeds the body of fundamental studies available in published literature. Much of this research is somewhat empirical. In addition to their interest for electro-metallurgy, fused salts have many analogies with fused silicates used as slags in bulk production of various metals. Their physical properties for applications as heat transfer fluids and bath fluids are also attracting increasing attention. All these considerations make any additions to published research, especially on the fundamental properties of fused salts, particularly welcome at the present time. Unfortunately, for many Western scientists researches published in Russian must still be regarded as not freely available until translations become accessible. The appearance of this collection prepared by the Consultants Bureau Inc. can therefore be described as very timely.

Translations necessarily suffer from certain defects in reproducing new knowledge. For example, the present collection includes nearly one hundred papers on melting and phase relationships for binary, ternary and quaternary mixtures of salts. The curves by means of which much of this information is recorded suffer in precision through the process of reproduction; such records may not be sufficiently accurate or clear for some purposes. However, it is very useful to know even the approximate shape of the diagrams in many cases.

In addition to a great bulk of work on phase diagrams, a limited number of the papers included give calculations on activity coefficients from electromotive force data, on the surface tension, density, viscosity and electrical conductivities of melts, on corrosion of metals by melts, and on slags.

Though discussions of basic theoretical considerations are included in some of the papers, the inspiration of much of this work appears to be technological. It constitutes an extensive body of factual knowledge about fused salts which should prove useful to those who do not have access to the original publications.

A. R. UBBELOHDE

\section{SOCIAL MEDICINE}

\section{The Teaching of Hygiene and Public Health in Europe}

A Review of Trends in Undergraduate and Postgraduate Education in Nineteen Countries. By Prof. F. Grundy and Prof. J. M. Mackintosh. (World Health Organization: Monograph Series, No. 34.) Pp. 254. (Geneva: World Health Organization; London: H.M. Stationery Office, 1957.) 15 Swiss franes ; 25s. ; 5 dollars.

$\mathrm{D}$ RING the past hundred years scientific methods have been applied widely in medical research and practice; in the same period the health of the population of the Western world has improved. But the improved health owes less to advances in medical science than to changes in the external environment, and a favourable trend in the standard of living. We are healthier than our ancestors not because of what happens when we are ill but because we do not become ill ; and we do not become ill not because of specific protective therapy but because we live in a healthier environment. In its preoccupation with the minutiae of diagnosis and the pathogenesis 